\title{
Completely local fully differential subtractions at NNLO
}

\section{Gábor Somogyi ${ }^{* \dagger}$}

MTA-DE Particle Physics Research Group H-4010 Debrecen, PO Box 105, Hungary

E-mail: gabor.somogyi@cern.ch

\section{Adam Kardos}

MTA-DE Particle Physics Research Group H-4010 Debrecen, PO Box 105, Hungary

E-mail: kardos.adamescience.unideb.hu

\section{Zoltán Trócsányi}

University of Debrecen and MTA-DE Particle Physics Research Group H-4010 Debrecen, PO

Box 105, Hungary

E-mail: zoltan.trocsanyidcern.ch

We present the CoLoRFulNNLO method for computing NNLO corrections to jet cross sections in perturbative QCD. We also discuss the application of our method to the computation of event shape observables in electron-positron annihilation into three jets and show predictions for the distribution of energy-energy correlation and oblateness, which were computed for the first time at this accuracy with this method.

Loops and Legs in Quantum Field Theory

24-29 April 2016

Leipzig, Germany

* Speaker.

${ }^{\dagger}$ This research was supported by the Hungarian Scientific Research Fund grant K-101482, the Post Doctoral Fellowship programme of the Hungarian Academy of Sciences and the Research Funding Program ARISTEIA, HOCTools (co-financed by the European Union (European Social Fund ESF) and Greek national funds through the Operational Program "Education and Lifelong Learning" of the National Strategic Reference Framework (NSRF)). 


\section{Introduction}

Any progress in the quest to understand the fundamental laws of Nature relies both on accurate measurements and on precise theoretical calculations. In particular, in order to fully exploit the physics potential of the LHC requires a detailed understanding of QCD, such that the experimental data can be confronted with highly precise theory predictions. One way in which the precision of the QCD predictions can be improved is by computing exact higher-order perturbative corrections to physical observables. However, when calculating QCD predictions beyond leading order (LO), one must face - in addition to other challenges - the problem of infrared (IR) singularities. Although these singularities cancel between the various contributions to IR-safe observables, nevertheless their presence in intermediate stages of the computation prevents the straightforward numerical implementation of QCD perturbation theory in specific processes.

Traditionally, at next-to-leading order (NLO) accuracy, a subtraction scheme was used to handle the problem of IR singularities. Indeed, the NLO correction to a generic $m$-jet observable $J$ is a sum of two pieces, the real emission and virtual contributions,

$$
\sigma^{\mathrm{NLO}}=\int_{m+1} \mathrm{~d} \sigma_{m+1}^{\mathrm{R}} J_{m+1}+\int_{m} \mathrm{~d} \sigma_{m}^{\mathrm{V}} J_{m},
$$

where $J_{n}$ denotes the value of $J$ evaluated on an $n$-parton final state. In eq. (1.1), both contributions are divergent in $d=4$ dimensions, however their sum is finite. The essential idea of a subtraction scheme is to use an approximate cross section, $\mathrm{d} \sigma_{m+1}^{\mathrm{R}, \mathrm{A}_{1}}$, to redistribute IR divergences between the real and virtual contributions,

$$
\sigma^{\mathrm{NLO}}=\int_{m+1}\left[\mathrm{~d} \sigma_{m+1}^{\mathrm{R}} J_{m+1}-\mathrm{d} \sigma_{m+1}^{\mathrm{R}, \mathrm{A}_{1}} J_{m}\right]_{d=4}+\int_{m}\left[\mathrm{~d} \sigma_{m}^{\mathrm{V}}+\int_{1} \mathrm{~d} \sigma_{m+1}^{\mathrm{R}, \mathrm{A}_{1}}\right]_{d=4} J_{m},
$$

such that both the $m+1$ and $m$ parton contributions become finite in $d=4$. Then the phase space integrals may be performed using standard numerical techniques in four dimensions. At NLO accuracy, several ways of constructing the approximate cross section are available [1]. These are all based on the known universal factorization properties of QCD matrix elements in IR limits and they share several key features:

1. The subtraction terms are defined completely explicitly for arbitrary processes and include all degrees of freedom (momentum, spin, color and flavor).

2. These algorithms produce fully differential results in four dimensions, so arbitrary detector cuts can be applied.

3. The subtraction terms are completely local over the phase space of real emission, including all color and spin correlations in IR limits.

4. The poles of the integrated subtraction terms can be computed analytically once and for all and can be shown to cancel the IR poles of the virtual contribution exactly.

However, despite the fact that the universal factorization formulae for QCD matrix elements in all IR limits relevant at next-to-next-to-leading order (NNLO) have been known for a while [2], it proved to be rather difficult to arrive at an NNLO computational scheme with all the desired 
properties in points 1-4. Hence several approaches were pursued [3] where one or more of these points were given up in favor of an easier construction. It is nevertheless possible to build a completely local subtraction scheme for fully differential calculations at NNLO accuracy starting from QCD IR-limit formulae. In this contribution, we present such a solution, the CoLoRFulNNLO (Completely Local subtRactions for Fully differential predictions at NNLO) method and apply our scheme to the computation of event shapes in electron-positron annihilation at NNLO accuracy.

\section{The CoLoRFulNNLO method}

Recall that the NNLO correction to some generic $m$-jet cross section specified by the jet function $J$ is a sum of the double real emission contribution (RR), the one-loop virtual contribution with one extra emission (RV) and the two-loop double virtual contribution (VV),

$$
\sigma^{\mathrm{NNLO}}=\int_{m+2} \mathrm{~d} \sigma_{m+2}^{\mathrm{RR}} J_{m+2}+\int_{m+1} \mathrm{~d} \sigma_{m+1}^{\mathrm{RV}} J_{m+1}+\int_{m} \mathrm{~d} \sigma_{m}^{\mathrm{VV}} J_{m}
$$

All of these contributions are separately divergent in $d=4$ dimensions due to the presence of IR singularities. As at NLO, the essential idea is to use approximate cross sections to rearrange these singularities between the three contributions in eq. (2.1). In the CoLoRFulNNLO method, this rearrangement is as follows,

$$
\sigma^{\mathrm{NNLO}}=\int_{m+2} \mathrm{~d} \sigma_{m+2}^{\mathrm{NNLO}}+\int_{m+1} \mathrm{~d} \sigma_{m+1}^{\mathrm{NNL}}+\int_{m} \mathrm{~d} \sigma_{m}^{\mathrm{NNLO}}
$$

where

$$
\begin{aligned}
& \mathrm{d} \sigma_{m+2}^{\mathrm{NNLO}}=\left\{\mathrm{d} \sigma_{m+2}^{\mathrm{RR}} J_{m+2}-\mathrm{d} \sigma_{m+2}^{\mathrm{RR}, \mathrm{A}_{2}} J_{m}-\left[\mathrm{d} \sigma_{m+2}^{\mathrm{RR}, \mathrm{A}_{1}} J_{m+1}-\mathrm{d} \sigma_{m+2}^{\mathrm{RR}, \mathrm{A}_{12}} J_{m}\right]\right\}_{d=4}, \\
& \mathrm{~d} \sigma_{m+1}^{\mathrm{NNLO}}=\left\{\left[\mathrm{d} \sigma_{m+1}^{\mathrm{RV}}+\int_{1} \mathrm{~d} \sigma_{m+2}^{\mathrm{RR}, \mathrm{A}_{1}}\right] J_{m+1}-\left[\mathrm{d} \sigma_{m+1}^{\mathrm{RV}, \mathrm{A}_{1}}+\left(\int_{1} \mathrm{~d} \sigma_{m+2}^{\mathrm{RR}, \mathrm{A}_{1}}\right)^{\mathrm{A}_{1}}\right] J_{m}\right\}_{d=4}, \\
& \mathrm{~d} \sigma_{m+1}^{\mathrm{NNLO}}=\left\{\mathrm{d} \sigma_{m}^{\mathrm{VV}}+\int_{2}\left[\mathrm{~d} \sigma_{m+2}^{\mathrm{RR}, \mathrm{A}_{2}}-\mathrm{d} \sigma_{m+2}^{\mathrm{RR}, \mathrm{A}_{12}}\right]+\int_{1}\left[\mathrm{~d} \sigma_{m+1}^{\mathrm{RV}, \mathrm{A}_{1}}+\left(\int_{1} \mathrm{~d} \sigma_{m+2}^{\mathrm{RR}, \mathrm{A}_{1}}\right)\right\}_{d=4}^{\mathrm{A}_{1}}\right]
\end{aligned}
$$

Equation (2.3) above includes the RR contribution which is singular whenever one or two partons become unresolved. From this we subtract the approximate cross section $\mathrm{d} \sigma_{m+2}^{\mathrm{RR}, \mathrm{A}_{2}}$ which regularizes the RR matrix element in all double unresolved limits. The difference however is still singular as one parton becomes unresolved and requires regularization. To that end, we subtract the approximate cross sections $\mathrm{d} \sigma_{m+2}^{\mathrm{RR}, \mathrm{A}_{1}}$ and $\mathrm{d} \sigma_{m+2}^{\mathrm{RR}, \mathrm{A}_{12}}$. It can be shown [4] that with these subtractions, the regularized RR contribution $\mathrm{d} \sigma_{m+2}^{\mathrm{NNLO}}$ is integrable over the $m+2$ parton phase space in four dimensions whenever $J$ defines an IR-safe observable.

Turning to eq. (2.4), it contains the RV contribution which describes the emission of one additional parton at one-loop. The real-virtual matrix element contains both explicit one-loop $\varepsilon$ poles as well as singularities when the additional parton is unresolved. The explicit IR singularities are the same as those of the single unresolved approximate cross section $\mathrm{d} \sigma_{m+2}^{\mathrm{RR}, \mathrm{A}_{1}}$ in integrated form and the $\varepsilon$ poles cancel in the sum of the first two terms in eq. (2.4). However, this sum is still singular in the single unresolved regions of phase space. In order to regularize these singularities, we subtract 
the approximate cross sections $\mathrm{d} \sigma_{m+1}^{\mathrm{RV}, \mathrm{A}_{1}}$ and $\left(\mathrm{d} \sigma_{m+2}^{\mathrm{RR}, \mathrm{A}_{1}}\right)^{\mathrm{A}_{1}}$. It can be shown [5] that the sum of these last two approximate cross sections does not contain explicit $\varepsilon$ poles and that the regularized RV contribution in eq. (2.4) is integrable over the $m+1$ parton phase space in four dimensions for any IR-safe jet function.

Finally, the VV contribution to the NNLO correction appears in eq. (2.5). This term does not lead to divergent phase space integrals (provided the observable is IR-safe), but it does contain explicit two-loop $\varepsilon$ poles. These are cancelled by the sum of the four integrated approximate cross sections which we have not yet added back to eq. (2.4), and which are shown in eq. (2.5).

The approximate cross sections that appear in eqs. (2.3)-(2.5) are defined explicitly by using the core concepts that underlie the general constructions at NLO accuracy: i) the known factorization properties of QCD matrix elements in the IR limits and ii) momentum mappings which lead to the exact factorization of phase space and thus allow for integrating over the unresolved momenta independently of the jet function. These cornerstones of the construction must be supplemented at NNLO by iii) a process-independent way of disentangling the overlapping singularities among both the various double unresolved limits as well as among the single and double unresolved limits. Clearly this third point is irrelevant at NLO where double unresolved singularities are not present.

As stated before, the factorization properties of QCD matrix elements in all IR limits relevant at NNLO have been known for some time. Even so, the implementation of the second and third points above poses a major challenge. In particular, the structure of overlaps of the various single and double unresolved limits is complicated and disentangling them is highly non-trivial. We presented a first solution to this problem at NNLO in [6], as well as a much more general solution, applicable in principle to any order in perturbation theory in [7]. We used these constructions to define approximate matrix elements which are free of double counting in all IR limits. However, as they stand, such approximate matrix elements cannot be used as subtraction terms because they are only well-defined in the strict soft and/or collinear limits. This is due to the fact that only in the strict limits do the momenta which enter the reduced matrix elements in the IR factorization formulae respect momentum conservation and the mass-shell conditions. Hence, in order to define true subtraction terms, we must specify precisely the momenta which enter the factorized matrix elements away from the strict limits. This is achieved by constructing mappings of sets of $m+2$ and $m+1$ momenta into sets of $m$ momenta in such a way that the mappings conserve momentum and the mass-shell conditions and have the correct limiting behavior in the IR limits. Furthermore, these mappings must lead to an exact factorization of phase space, and they must respect the delicate structure of cancellations among the various approximate matrix elements in each IR singular region. We presented such mappings along with the precise definitions of all double real emission approximate cross sections that appear in eq. (2.3) in [4]. The exact definitions of all approximate cross sections relevant to regularizing the real-virtual contribution in eq. (2.4) were given in [5].

We stress that our construction leads to completely local subtraction terms that properly take all color and spin correlations into account in all singular limits. Hence, the $m+2$ and $m+1$ parton phase space integrals in eqs. (2.3) and (2.4) converge in $d=4$ dimensions and may be computed with whatever numerical procedure is most convenient.

Finally, we must integrate the approximate cross sections over the phase space of the unresolved emission(s), and combine their integrated forms with the VV contribution as shown in 
eq. (2.5). Owing to the simultaneous factorization of both the phase space and approximate matrix elements in all IR limits, this integration can be performed once and for all independently of the specific process or observable. After performing the integration, as well as summing over the color and flavor of the unobserved parton(s) - denoted symbolically by $\int_{1}$ and $\int_{2}$ in eqs. (2.4) and (2.5) for single and double unresolved emission, respectively - each integrated approximate cross section can be written as a product (in color space) of a lower point cross section with a singular (in $\varepsilon$ ) insertion operator.

Computing the singular insertion operators explicitly is a laborious task that involves the evaluation of many cumbersome multi-dimensional phase space integrals [8]. We have expanded all of these integrals in $\varepsilon$ and computed the poles of these Laurent expansions fully analytically. We have also obtained analytic expressions for all finite terms that diverge logarithmically on the boundary of the phase space, while the remaining finite regular terms were computed numerically. As the poles of all integrated counterterms are known analytically, we can explicitly demonstrate the cancellation of the $\varepsilon$ poles in the regularized double virtual contribution of eq. (2.5). We have shown this explicitly for the case of $m=2$ in [9], while the case of $m=3$ was discussed in [10].

\section{Event shapes in electron-positron annihilation}

As an application of our method, we have computed predictions for event shape observables in electron-positron annihilation at NNLO accuracy. By way of illustration, we present in figure 1 our results for the physical distributions as well as the perturbative NNLO coefficients for thrust, $C$-parameter and the two-to-three jet transition variable $y_{23}$ in the Durham jet clustering algorithm. The perturbative coefficients are defined by normalizing the result to the leading order cross section for $e^{+} e^{-} \rightarrow$ hadrons, $\sigma_{0}$,

$$
\frac{1}{\sigma_{0}} \frac{\mathrm{d} \sigma}{\mathrm{d} O}=\frac{\alpha_{\mathrm{s}}}{2 \pi} A(O)+\left(\frac{\alpha_{\mathrm{s}}}{2 \pi}\right)^{2} B(O)+\left(\frac{\alpha_{\mathrm{s}}}{2 \pi}\right)^{3} C(O)+\mathrm{O}\left(\alpha_{\mathrm{s}}^{4}\right) .
$$

These particular event shapes have been computed at NNLO accuracy before in [11] and [12]. Therefore in figure 1 we present also the comparison of our predictions to the previously published ones $^{1}$. We find quite good agreement with the predictions of SW and reasonably good agreement with those of GGGH, except for very small and large values of the event shapes, however the precise comparison to the GGGH predictions is hampered by the somewhat large integration uncertainties and bin-to-bin fluctuations of those results. Regarding the deviations of the predictions for large values of event shapes, we note that these appear in regions where the contributions from three particle final states vanish (e.g., $\tau=1-T>1 / 3$ and $C_{\mathrm{par}}>3 / 4$ ). Thus the distributions are determined by NLO corrections to four parton final states which have been known for a long time [13] and can be computed also with modern automated tools. We have checked that our predictions agree perfectly with those of MadGraph5_aMC@NLO [14] in these regions. We have also checked that our results agree with the resummed predictions of SCET [15] expanded to $O\left(\alpha_{\mathrm{s}}^{3}\right)$ for small values of event shapes. Finally, we note that we observe a very good numerical convergence of our method at NNLO. The relative statistical uncertainties of our NNLO predictions are shown as gray

\footnotetext{
${ }^{1}$ In the figure we compare to updated but unpublished results from both [11] and [12]. We are grateful to G. Heinrich and S. Weinzierl for providing these predictions for us.
} 

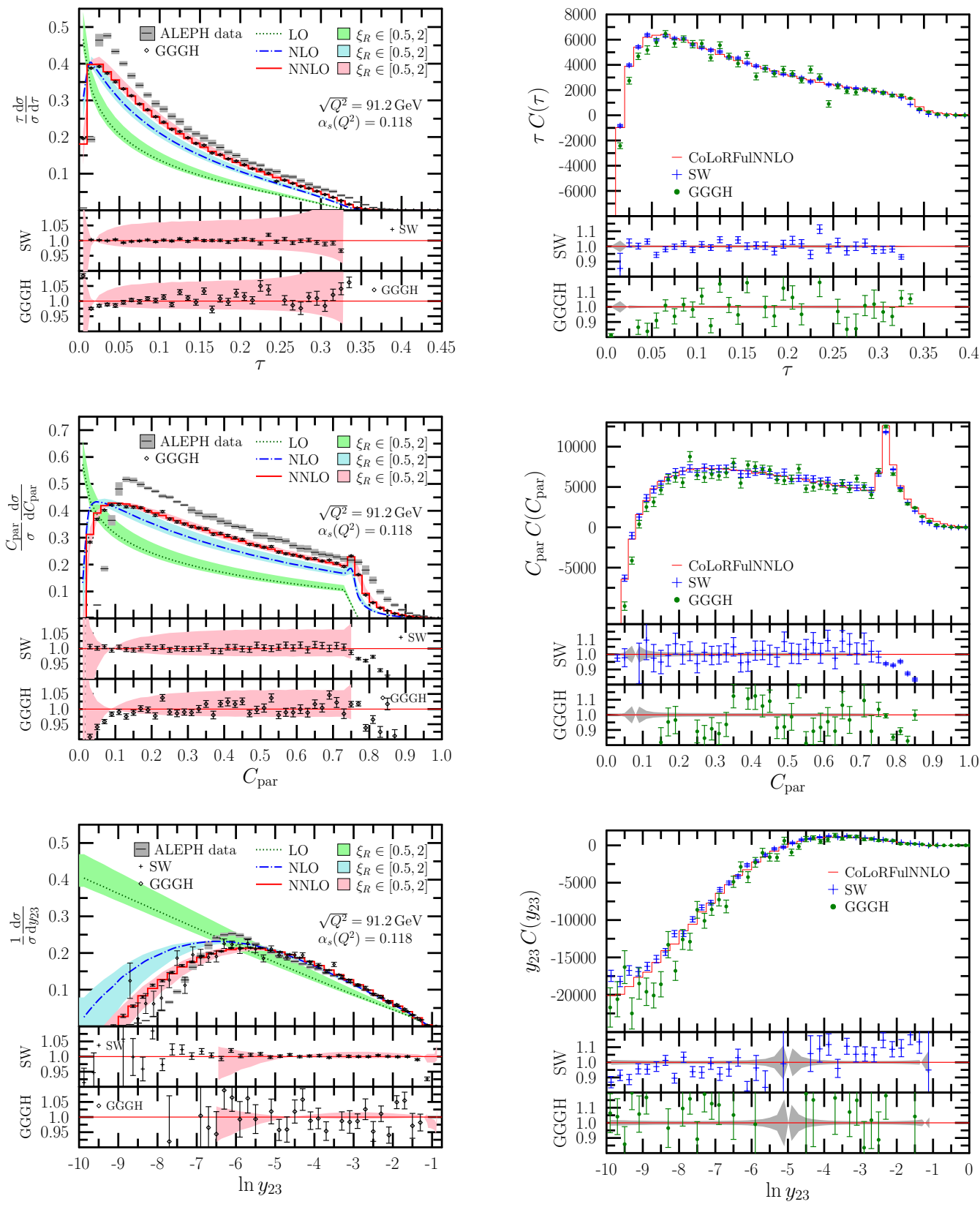

Figure 1: Left: perturbative predictions for thrust, $C$-parameter and the two-to-three jet transition variable $y_{23}$ in the Durham jet clustering algorithm at LO, NLO and NNLO accuracy. The bands represent the dependence on the renormalization scale corresponding to the range $\xi_{R} \equiv \mu / \sqrt{Q^{2}} \in[0.5,2]$. The lower panels show the ratio of the predictions of [11] (GGGH) and [12] (SW) to CoLoRFulNNLO, the red bands show the relative scale uncertainty. Right: predictions for the $O C(O)$ coefficients for the same observables. The lower panels are as before, but the gray bands show the relative statistical uncertainties of our predictions due to Monte Carlo integrations. 
bands on the lower panels of the right hand side plots in figure 1, while the scattered error bars represent the statistical uncertainties of the other two predictions.

Our method and code allow us to compute any IR-safe observable in $e^{+} e^{-} \rightarrow 3$ jets at NNLO accuracy. Here we show two examples of event shapes that have not been computed to this accuracy previously: energy-energy correlation (EEC) and oblateness. In figure 2 we present our physical predictions for these observables at LO, NLO and NNLO accuracy. We again observe a very
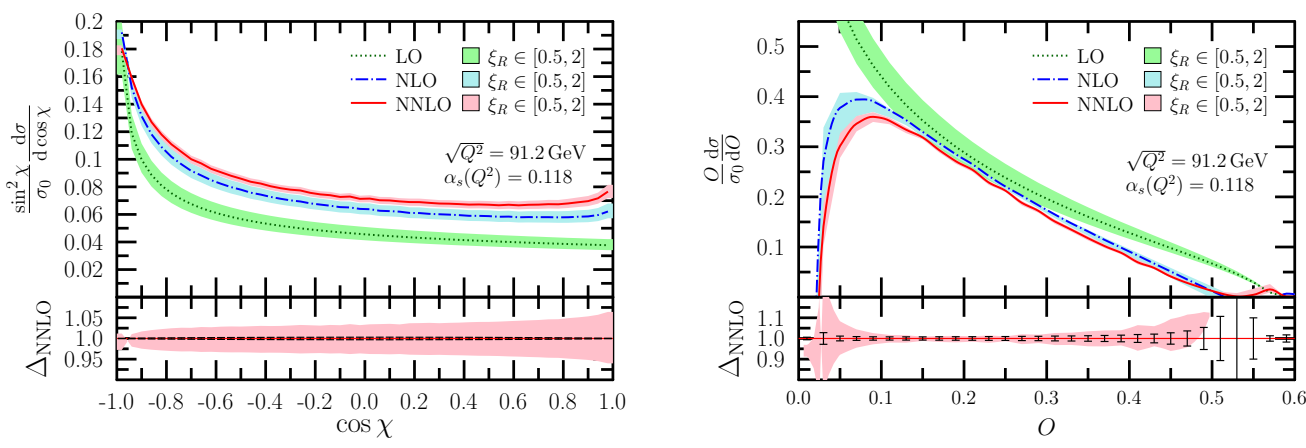

Figure 2: Physical predictions for energy-energy correlation (left) and oblateness (right) at LO, NLO and NNLO accuracy. Bands represent the uncertainty due to scale variation in the range $\xi_{R} \in[0.5,2]$.

good numerical convergence of our code: the relative statistical uncertainties due to Monte Carlo integrations are shown as error bars around the line at one on the lower panels of the plots in the figure. In order to be able to better appreciate the impact of the NNLO corrections, we present in
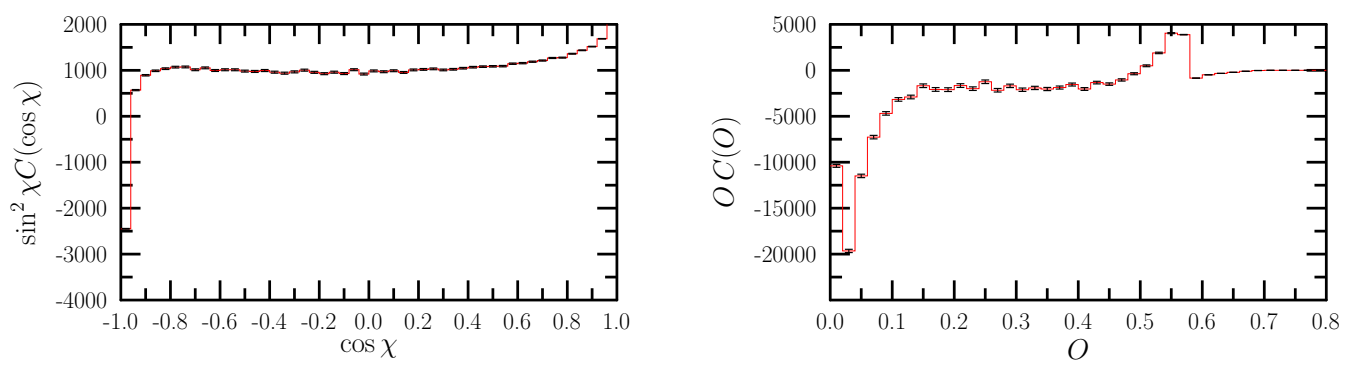

Figure 3: Distributions of the NNLO coefficients for energy-energy correlation (left) and oblateness (right). The error bars represent the statistical uncertainties of the Monte Carlo integrations.

figure 3 the distributions of the NNLO coefficients for EEC and oblateness. We observe that the NNLO corrections enhance the EEC distribution almost uniformly, while they slightly lower and also slightly modify the shape of the oblateness distribution.

\section{Conclusions}

We presented the CoLoRFulNNLO method for computing jet cross sections at NNLO accuracy. We also discussed the application of our method to the computation of event shape observ- 
ables in electron-positron annihilation into three jets. We found good agreement with previous results when available. We also showed predictions for the distributions of energy-energy correlation and oblateness at NNLO, which were obtained for the first time with this method. We found a very good numerical convergence of our method and code for all observables considered. The CoLoRFulNNLO method is fully worked out for processes with colorless initial states. The generalization to hadronic initial states is work in progress.

\section{References}

[1] S. Frixione, Z. Kunszt and A. Signer, Nucl. Phys. B 467 (1996) 399 [hep-ph/9512328]; S. Catani and M. H. Seymour, Nucl. Phys. B 485 (1997) 291 Erratum: [Nucl. Phys. B 510 (1998) 503] [hep-ph/9605323]; Z. Nagy and Z. Trócsányi, Nucl. Phys. B 486 (1997) 189 [hep-ph/9610498]; G. Somogyi, JHEP 0905 (2009) 016 [arXiv:0903.1218 [hep-ph]].

[2] See e.g., S. Catani and M. Grazzini, Nucl. Phys. B 570 (2000) 287 [hep-ph/9908523]; Z. Bern, V. Del Duca, W. B. Kilgore and C. R. Schmidt, Phys. Rev. D 60 (1999) 116001 [hep-ph/9903516] and references therein.

[3] A. Gehrmann-De Ridder, T. Gehrmann and E. W. N. Glover, JHEP 0509 (2005) 056 [hep-ph/0505111]; S. Catani and M. Grazzini, Phys. Rev. Lett. 98 (2007) 222002 [hep-ph/0703012]; M. Czakon, Phys. Lett. B 693 (2010) 259 [arXiv:1005.0274 [hep-ph]]; R. Boughezal, C. Focke, X. Liu and F. Petriello, Phys. Rev. Lett. 115 (2015) no.6, 062002 [arXiv:1504.02131 [hep-ph]]; J. Gaunt, M. Stahlhofen, F. J. Tackmann and J. R. Walsh, JHEP 1509 (2015) 058 [arXiv:1505.04794 [hep-ph]].

[4] G. Somogyi, Z. Trócsányi and V. Del Duca, JHEP 0701 (2007) 070 [hep-ph/0609042].

[5] G. Somogyi and Z. Trócsányi, JHEP 0701 (2007) 052 [hep-ph/0609043].

[6] G. Somogyi, Z. Trócsányi and V. Del Duca, JHEP 0506 (2005) 024 [hep-ph/0502226].

[7] Z. Nagy, G. Somogyi and Z. Trócsányi, hep-ph/0702273 [HEP-PH].

[8] See G. Somogyi, JHEP 1304 (2013) 010 [arXiv:1301.3919 [hep-ph]] and references therein.

[9] V. Del Duca, C. Duhr, G. Somogyi, F. Tramontano and Z. Trócsányi, JHEP 1504 (2015) 036 [arXiv:1501.07226 [hep-ph]].

[10] V. Del Duca, C. Duhr, A. Kardos, G. Somogyi and Z. Trócsányi, arXiv:1603.08927 [hep-ph]; V. Del Duca, C. Duhr, A. Kardos, G. Somogyi, Z. Szőr, Z. Trócsányi and Z. Tulipánt, arXiv:1606.03453 [hep-ph].

[11] A. Gehrmann-De Ridder, T. Gehrmann, E. W. N. Glover and G. Heinrich, JHEP 0712 (2007) 094 [arXiv:0711.4711 [hep-ph]].

[12] S. Weinzierl, JHEP 0906 (2009) 041 [arXiv:0904.1077 [hep-ph]].

[13] A. Signer and L. J. Dixon, Phys. Rev. Lett. 78 (1997) 811 [hep-ph/9609460]; Z. Nagy and Z. Trócsányi, Phys. Rev. Lett. 79 (1997) 3604 [hep-ph/9707309].

[14] J. Alwall et al., JHEP 1407 (2014) 079 [arXiv:1405.0301 [hep-ph]].

[15] T. Becher and M. D. Schwartz, JHEP 0807 (2008) 034 doi:10.1088/1126-6708/2008/07/034 [arXiv:0803.0342 [hep-ph]]; Y. T. Chien and M. D. Schwartz, JHEP 1008 (2010) 058 doi:10.1007/JHEP08(2010)058 [arXiv:1005.1644 [hep-ph]]; A. H. Hoang, D. W. Kolodrubetz, V. Mateu and I. W. Stewart, Phys. Rev. D 91 (2015) no.9, 094017 doi:10.1103/PhysRevD.91.094017 [arXiv:1411.6633 [hep-ph]]. 